\title{
LASER-ASSISTED ELECTRON-ATOM COLLISIONS
}

\author{
C. J. JOACHAIN \\ Physique Théorique, Université Libre de Bruxelles, Belgium
}

\begin{abstract}
The theoretical methods which have been developed to analyze laser-assisted electron-atom collisions are reviewed. Firstly, the scattering of an electron by a potential in the presence of a laser field is considered. The analysis is then generalized to laser-assisted collisions of electrons with "real" atoms having an internal structure. Two methods are discussed: a semi-perturbative approach suitable for fast incident electrons and a fully non-perturbative theory-the R-matrix-Floquet method-which is applicable to the case of slow incident electrons. In particular it is shown how the dressing of the atomic states by the laser field can affect the collision cross sections.
\end{abstract}

KEY WORDS: Multiphoton processes, laser-assisted scattering.

\section{INTRODUCTION}

The study of electron-atom collisions in the presence of a laser field has attracted a great deal of attention in recent years, not only because of the importance of these processes in applied areas (such as plasma heating) but also in view of their interest in fundamental atomic collision theory. In particular, they allow the observation of multiphoton processes at relatively moderate laser field intensities and give rise to a number of interesting new effects with respect to field-free collisions. Reviews of various aspects of laser-assisted electron-atom collisions can be found in the references. ${ }^{1-3}$

The theoretical study of electron-atom collisions in the presence of a laser field is in general very complex, since in addition to the difficulties associated with the treatment of field-free electron-atom scattering, the presence of the laser field introduces new parameters (such as the laser frequency, intensity, polarization and statistics) which can influence the collisions. It is therefore of interest to begin the theoretical analysis by considering the simpler problem of the scattering of an electron by a potential, in the presence of a laser field. Section 2 is devoted to a survey of this subject. In Section 3 we generalize the study to laser-assisted collisions of electrons with "real" atoms, having an internal structure. In what follows we shall assume that the laser field is treated classically as a monochromatic, monomode, linearly polarized and spatially homogeneous electric field. The rôle played by fluctuations of the laser field will not be discussed here; it is examined for example in Ref. (2). Atomic units will be used throughout. 


\section{POTENTIAL SCATTERING}

We begin by considering the scattering of an electron by a potential $V(\mathbf{r})$ in the presence of a laser field described by the electric field $\varepsilon(t)=\varepsilon_{0}$ sin $\omega t$. The wave function of a "free" electron embedded in this field is given, in the velocity gauge, by the Volkov wave function

$$
\chi_{\mathbf{k}}(\mathbf{r}, t)=(2 \pi)^{-3 / 2} \exp \left(i \mathbf{k} \cdot \mathbf{r}-i \mathbf{k} \cdot \alpha_{0} \sin \omega t-i E_{k} t\right)
$$

where $\mathbf{k}$ is the electron momentum, $E_{k}=k^{2} / 2$ its kinetic energy and $\alpha_{0}=\varepsilon_{0} / \omega^{2}$. If $\mathbf{k}_{i}$ and $\mathbf{k}_{f}$ denote the electron momentum in the initial and final state, respectively, the $S$-matrix element for a transition $\mathbf{k}_{i} \rightarrow \mathbf{k}_{f}$, in the presence of the laser field is given by

$$
S_{\mathbf{k}_{f}, \mathbf{k}_{i}}=(2 \pi)^{-1} i \sum_{l=-\infty}^{+\infty} \delta\left(E_{k_{f}}-E_{k_{i}}-l \omega\right) f^{l}\left(\mathbf{k}_{f}, \mathbf{k}_{i}\right)
$$

where $f^{l}\left(\mathbf{k}_{f}, \mathbf{k}_{i}\right)$ is the scattering amplitude for the transition $\mathbf{k}_{i} \rightarrow \mathbf{k}_{f}$ accompanied by the transfer of $l$ photons. We remark that positive integer values of $l$ correspond to photon absorption (inverse bresmsstrahlung), negative integer ones to photon emission (stimulated bremsstrahlung) and $l=0$ to elastic scattering modified by the presence of the laser field.

The evaluation of the scattering amplitudes $f^{l}\left(\mathbf{k}_{f}, \mathbf{k}_{i}\right)$ and of the corresponding differential cross sections is in general a difficult task. However, in some limiting cases, important simplifications occur. In particular, in the low frequency (soft photon) limit, such that $\hbar \omega \ll k_{i}^{2} / 2$, Kroll and Watson ${ }^{4}$ have shown that the cross section for the scattering process $\mathbf{k}_{i} \rightarrow \mathbf{k}_{f}$ accompanied by the transfer of $l$ photons is given by

$$
\frac{d \sigma^{l}}{d \Omega}=\frac{k_{f}(l)}{k_{i}} J_{l}^{2}\left(\mathbf{\Delta} \cdot \alpha_{0}\right) \frac{d \sigma}{d \Omega}\left(\mathbf{k}_{f}^{*}, \mathbf{k}_{i}^{*}\right)
$$

where $k_{f}^{2}(l)=k_{i}^{2}+2 l \omega, \Delta=\mathbf{k}_{i}-\mathbf{k}_{f}$ is the momentum transfer, $J_{l}$ is an ordinary Bessel function of order $l, d \sigma\left(\mathbf{k}_{f}^{*}, \mathbf{k}_{i}^{*}\right) / d \Omega$ is the field free differential cross section corresponding to the transition $\mathbf{k}_{i}^{*} \rightarrow \mathbf{k}_{f}^{*}$ and $\mathbf{k}_{j}^{*}(j=i, f)$ are shifted momenta such that $\mathbf{k}_{j}^{*}=\mathbf{k}_{j}$ $+l \omega \alpha_{0} /\left(\Delta \cdot \alpha_{0}\right)$. It is worth stressing that the Kroll-Watson result is non-perturbative both in the laser-projectile interaction and in the projectile-target interaction potential $V(\mathbf{r})$. If the latter interaction can be treated by using first order perturbation theory, then in Eq. (3) the quantity $d \sigma\left(\mathbf{k}_{f}^{*}, \mathbf{k}_{i}^{*}\right) / d \Omega$ can be replaced by the field-free first Born differential cross section $d \sigma^{B 1} / d \Omega$ and the Kroll-Watson formula reduces to the first Born result obtained previously by Bunkin and Fedorov. ${ }^{5}$ We also remark that when scattering resonances exist in the absence of the laser field the Kroll-Watson formula must be modified. ${ }^{6-8}$

Another limiting case in which simplifications occur is that of low-energy electrons scattered by a potential in the presence of a high-intensity, high frequency laser field. This case has been investigated by Gavrila et al ${ }^{9-12}$ By applying the Kramers space translation $^{13}$ and using the Floquet theory of linear differential equations with periodic coefficients, Gavrila et al. showed that in the high-intensity, high-frequency limit, and at sufficiently low electron energies, only modified slastic scattering in the presence of the laser field $(l=0)$ takes place appreciably. They performed a detailed 
study of modified Coulomb scattering and found large deviations from the Rutherford cross sections in the non-perturbative regime. ${ }^{12}$ The Coulomb scattering of low energy electrons by protons in the presence of a high-frequency, strong laser field has also been investigated along similar lines by Dimou and Faisal. ${ }^{14}$ They found that the field-modified elastic scattering $(l=0)$ and inverse bremsstrahlung cross sections are dominated by capture-escape resonances.

\section{ELECTRON-ATOM COLLISIONS}

Let us now consider collisions of electrons with "real" atoms- - having an internal structure-in the presence of a laser field. Three types of interactions must be taken into account. First, the interaction between the unbound electron and the target atom takes place, as in the field-free case. Second, the laser field interacts with the unbound electron. Third, the laser field interacts with the target atom and hence "dresses" the atomic target states.

We shall first discuss a semi-perturbative theory which we have developed over the last few years. ${ }^{1,2,15-20} \mathrm{We}$ consider the case for which the incident electrons are fast $\left(E_{k i} \geq 100 \mathrm{eV}\right)$ so that the electron-atom interaction can be treated perturbatively by using the Born series. ${ }^{21}$ The interaction between the laser field $\varepsilon(t)=\varepsilon_{0} \sin \omega t$ and the projectile electron can be taken into account exactly, as in the case of potential scattering, by using a Volkov wave function. On the other hand, the laser-atom interaction can be treated by using first order time-dependent perturbation theory, provided that the electric field strength $\varepsilon_{0}$ remains small with respect to the atomic unit of electric field strength $e / a_{0}^{2} \simeq 5 \times 10^{9} \mathrm{~V} \mathrm{~cm}^{-1}$. For direct (no electron exchange) laser-assisted elastic or inelastic collisions $\left(\mathbf{k}_{i}, i\right) \rightarrow\left(\mathbf{k}_{f}, f\right)$, in which the index $i$ refers to the initial state of the atom and the index $f$ to its final state, the $S$-matrix element can be written in the form

$$
S_{\mathbf{k}_{f} f ; \mathbf{k} i, i}=(2 \pi)^{-1} i \sum_{l=-\infty}^{+\infty} \delta\left(E_{k_{f}}-E_{k_{l}}-E_{f}-E_{i}-l \omega\right) f_{f i}^{l}\left(\mathbf{k}_{f}, \mathbf{k}_{i}\right)
$$

where $f_{f i}^{l}\left(\mathbf{k}_{f}, \mathbf{k}_{i}\right)$ is the direct scattering amplitude for the collision process $\left(\mathbf{k}_{i}, i\right) \rightarrow$ $\left(\mathbf{k}_{f}, f\right)$ accompanied by the transfer of $l$ photons. Using for the perturbative treatment of the electron-atom interaction the third order eikonal-Born series (EBS) method of Byron and Joachain, ${ }^{21-23}$ we have made a detailed analysis of the direct scattering amplitude $f_{f i}^{l}\left(\mathbf{k}_{f}, \mathbf{k}_{i}\right)$ and of the corresponding exchange scattering amplitude $g_{f i}^{l}\left(\mathbf{k}_{f}, \mathbf{k}_{i}\right)$ for various laser-assisted elastic and inelastic electron-atom collisions. ${ }^{1,2,15-18}$ We find that for electric field strengths $\varepsilon_{0}$ which are large by laboratory standards but nevertheless much smaller than the atomic unit, the dressing of the target by the laser field can lead to dramatic modifications of the angular distributions. More recently, we have extended our calculations to laser-assisted $(e, 2 e)$ reactions ${ }^{19.20}$ where we also find that target dressing effects are responsible for important modifications of the angular distributions.

Two other important effects which we have found using the foregoing analysis are (i) the appearance in the cross sections of asymmetries between the absorption and 
the emission of a given number of photons and (ii) the appearance of new resonant structures in the cross sections when the laser photon energy matches the energy difference between the initial or final state of the atom and an intermediate atomic state. ${ }^{16-20}$

The semi-perturbative method described above can be imporved by using the Floquet theory to construct dressed target states in a non-perturbative way. This is particularly important in resonant cases. In particular, using the Floquet theory together with Feshbach projection operators, Francken and Joanchain ${ }^{24}$ have analyzed the electron impact excitation of the $2^{1} S$ and $2^{1} P$ states of helium in the presence of a laser field which resonantly couples the two excited final states.

To conclude, we shall now briefly describe a completely non-perturbative theory, the R-matrix-Floquet method, ${ }^{25,26}$ which we have proposed to treat both the multiphoton ionization of atoms and laser-assisted electron-atom collisions. We consider an atomic system composed of a nucleus of atomic number $Z$ and $(N+1)$ electrons, in a laser field which, as stated in Section 1, is assumed to be monochromatic, monomode, linearly polarized and spatially homogeneous.

In accordance with the R-matrix method, we subdivide configuration space into two regions, as shown in Figure 1 . The internal region is defined by the condition that the radial coordinates $r_{i}$ of all $N+1$ electrons are such that $r_{i} \leq a(i=1,2, \ldots N+$ $1)$, where the sphere of radius $a$ envelops the charge distribution of the target atom states retained in the calculation. The external region is defined by the condition that one of the $(N+1)$ electrons lies on or outside this sphere and the remaining $N$ electrons are confirmed within this sphere. The Schrödinger equation is then solved in both regions by using the Floquet method. It should be noted that in each region the most appropriate form of the laser-atomic system interaction Hamiltonian can be chosen by performing unitary transformations on the wave function. Thus, as indicated in Figure 1, we use the length gauge in the internal region and the velocity gauge in the external region. At some large radius $a^{\prime} \gg a$ it is sometimes convenient to further transform the equations to the Kramers acceleration frame, ${ }^{13}$ where simpler asymptotic boundary conditions can be defined. We also remark that in the internal region the spectrum is entirely discrete, so that the standard Hermitian Floquet theory can be used. By matching the internal and external solutions on the sphere of radius $a$, and using appropriate boundary conditions, one can then extract

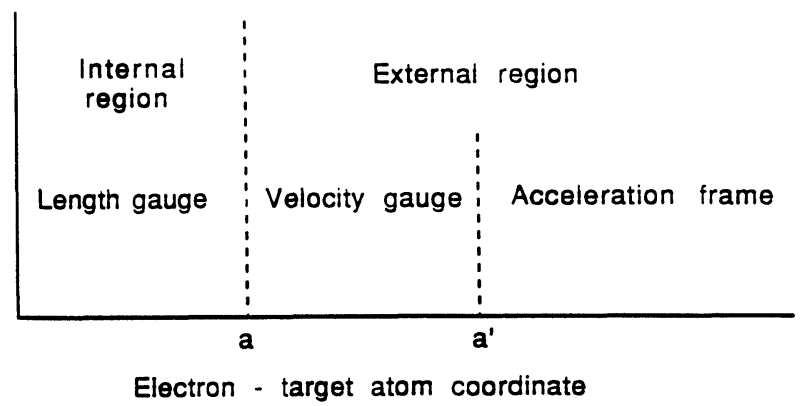

Figure 1 Partition of configuration space in the R-matrix-Floquet theory. 
the theoretical quantities to be compared with experiment. The R-matrix Floquet method has already been applied successfully to the calculation of multiphoton ionization rates for a one-dimensional model ${ }^{25,26}$ These calculations are now being extended to multiphoton ionization and laser-assisted electron-atom collisions for various atomic targets of experimental interest.

\section{References}

1. C. J. Joachain, in "Fundamentals of Laser Interactions," ed. by F. Ehlotzky (Springer-Verlag, Berlin, 1985), p. 37.

2. P. Francken and C. J. Joachain, J. Opt. Soc. Am. B 7, 554 (1990).

3. N. J. Mason, Contemp. Phys. 30, 449 (1989).

4. N. M. Kroll and K. M. Watson, Phys. Rev. A 8, 804 (1973).

5. F. V. Bunkin and M. V. Fedorov, Sov. Phys. JETP 22, 844 (1966).

6. H. Krüger and C. Jung, Phys. Rev. A 17, 1706 (1978).

7. M. H. Mittleman, Phys. Rev. A 20, 1965 (1979).

8. L. Rosenberg, Phys. Rev. A 23, 2283 (1981).

9. M. Gavrila and J. Z. Kaminski, Phys. Rev. Lett. 52, 613 (1984).

10. M. J. Offerhaus, J. Z. Kaminski and M. Gavrila, Phys. Lett. 112A, 151 (1985).

11. M. Gavrila, M. J. Offerhaus and J. Z. Kaminski, Phys. Lett. 118A, 331 (1986).

12. J van de Ree, J. Z. Kaminski and M. Gavrila, Phys. Rev. A 37, 4536 (1988).

13. H. A. Kramers, "Collected Scientific Papers" (North Holland, Amsterdam, 1956), p. 262.

14. L. Dimou and F. H. M. Faisal, Phys. Rev. Lett. 59, 872 (1987).

15. F. W. Byron Jr., and C. J. Joachain, J. Phys. B 17, L295 (1984).

16. P. Francken and C. J. Joachain, Phys. Rev. A 35, 1590 (1987).

17. F. W. Byron Jr., P. Francken and C. J. Joachain, J. Phys. B 20, 5487 (1987).

18. P. Francken, Y. Attaourti and C. J. Joachain, Phys. Rev. A 38, 1785 (1988).

19. C. J. Joachain, P. Francken, A. Maquet, P. Martin and V. Véniard, Phys. Rev. Lett. 61, 165 (1988).

20. P. Martin, V. Véniard, A. Maquet, P. Francken and C. J. Joachain, Phys. Rev. A 39, 6178 (1989).

21. C. J. Joachain, "Quantum Collision Theory" (North Holland, Amsterdam, 3d ed., 1983).

22. F. W. Byron Jr. and C. J. Joachain, Phys. Rev. A 8, 1267 (1973).

23. F. W. Byron Jr. and C. J. Joachain, Phys. Reports 34, 233 (1977).

24. P. Francken and C. J. Joachain, Physical Review A 41, 3770 (1990).

25. P. G. Burke, P. Francken and C. J. Joachain, Europhys. Lett. 13, 617 (1990).

26. P. G. Burke, P. Francken and C. J. Joachain, J. Phys. B 24, 761 (1991). 